\title{
comportement géotechnique des résidus urbains
}

\author{
par \\ G. Cartier \\ L.C.P.C., Paris
}

RESUME - Les projets de construction sur zones de décharges d'ordures ménagères se heurtent bien souvent à l'évaluation de la canacité portante, des tassements et des phénomènes liés à l'activité biochimique. On fait donc ici le point des nroblèmes rencontrés et de la méthodologie existante. Des résultats obtenus sur deux sites expérimentaux par les Laboratoires des Ponts et Chaussées sont analysés et comparés aux valeurs proposées dans la littérature spécialisée.

\section{INTRODUCTION}

Le stockage des résidus modernes, dont la production crût de façon quasi-exnonentielle, est devenu un problème économique majeur : en 1977 les Etats-Unis produisaient $10^{9}$ à $3 \times 10^{9}$ tonnes de déchets annuellement, soit une moyenne de 10 à $30 \mathrm{~kg}$ par personne et par jour. Parmi cette quantité, 1 à $2 \mathrm{~kg}$ sont imputables à la consommation individuelle journalière, ce qui est à comparer aux valeurs fournies par la Direction de la Prévention des Pollutions pour la France, soit 0,6 à 1 kg par habitant et par jour en 1979.

Malgré l'existence de traitements des déchets dès la production (recyclaçe, incinération, pyrolyse, fermentation méthanique, compactage, ...), une part importante des sous-produits est mise en décharge dans des zones dont on pourrait souvent tirer profit dans le cadre de zones d'aménagement ou de projets routiers, Les possibilités de réutilisation des déchets, notamment comme matériau de fondation, sont toutefois encore assez mal connues (Cartier et al., 1981).

Parmi les résidus non uniquement ménagers, les matériaux divers souvent déversés sans contrôle pour combler d'anciennes ballastières ne posent généralement pas de problèmes insurmontables de constructibilité. Les tassements sont assez rapides et les propriétés mécaniques peuvent être améliorées, notamment par oréchargement (Cudennec, 1981). Les déchets et sous-produits industriels, par contre, sont très particuliers selon leur nature et leur provenance mais, même s'ils doivent être caractérisés dans le cadre d'études spécifiques, ils sont toutefois souvent homogènes et bien connus.

Les déchets ménagers, par contre, ont les caractéristiques médiocres des sol's très mous mais sont beaucoup plus hétérogènes surtout dans les anciennes décharges urbaines non contrôlées. De plus, ils comportent une proportion imnortante d'éléments nutrescibles actifs chimiquement et biologiquement et leurs caractéristiques sont donc sujettes à des variations très importantes. On connait toutefois de nombreux exembles de constructions sur décharges : quartiers d'habitation, complexes de loisirs, grands immeubles, centres commerciaux et même pistes d'aéronorts (Aéroport de La Guardia à New York, par exemple).

La synthèse bibliographique présentée ici permet de dresser un bilan des principaux problèmes que 1 'on rencontre quand on doit construire sur des résidus ménagers :

- difficultés de caractérisation du matériau,

- faible capacité portante,

- importance des tassements, notamment secondaires,

- choix des dispositions constructives à retenir pour les projets. 
On compare également ces résultats avec ceux obtenus par les Laboratoires des Ponts et Chaussées lors du suivi de deux sites expérimentaux qui couvrent la gamme des décharges de résidus urbains : la décharge municipale de Roanne et la décharge contrôlée et moderne d'Arnouville (fig. 1 et 2).

\section{CARACTERISTIOUES DES DECHETS MENAGERS}

Les résidus de l'usage domestique sont traditionnellement stockés dans des décharges municipales qui peuvent également recevoir la collecte de déchets variés. Le caractère parfois sauvage de cetyoe de décharge lié aux problèmes de pollution en cours d'épandage encourage de plus en plus souvent les municipalités à avoir recours à des installations plus rationnelles permettant parfois de réhabiliter des sites dégradés (carrières par exemple). La décharge contrôlée d'Arnouville (fig. 1) est reorésentative de ce type de site où, à l'intérieur de casiers successifs, on rénand les ordures en couches de 2 à $2,50 \mathrm{~m}$ d'épaisseur recouvertes journellement par un matériau inerte. Ces opérations sont généralement assorties d'un broyage et d'un compactage.

Le tableau I donne un aperçu de la composition de différentes décharges. Le comportement global du mátériau dépend essentiellement du comportement des constituants qui, suivant leur nature, peuvent fermenter, pourrir, brûler, se corroder ou s'écraser. Ces différents processus qui contribuent aux opérations de décombosition sont liés à la teneur en eau qui est élevée dans les matières végétales et animales, ce qui corresnond à une valeur globale moyenne de 10 à 50 \%. La densité est également un paramètre majeur du combortement des résidus mais sa détermination reste délicate : on estime généralement
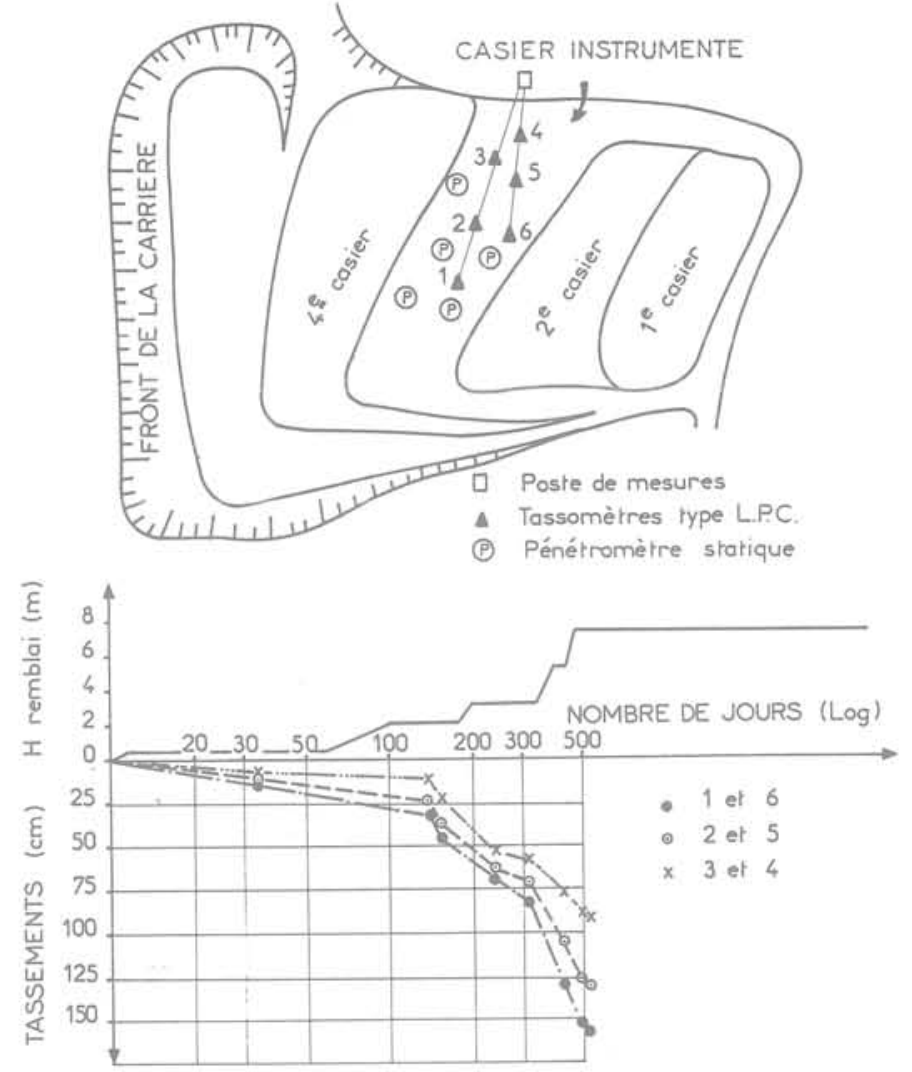

Fig. 1 La décharge contrôlée d'Arnouville : Instrumentation et résultats

que le poids volumique peut varier entre $1,5-3 \mathrm{KN} / \mathrm{m} 3$ et $3-6,5 \mathrm{KN} / \mathrm{m} 3$ suivant le degré de compacité, un compactage très énergique pouvant augmenter ces valeurs jusqu'à 10 $\mathrm{KN} / \mathrm{m} 3$. L'indice des vides varie dans une fourchette très large suivant que les déchets sont bien compactés $\left(e_{0}=2\right)$ ou non $\left(e_{0}=15\right)$.

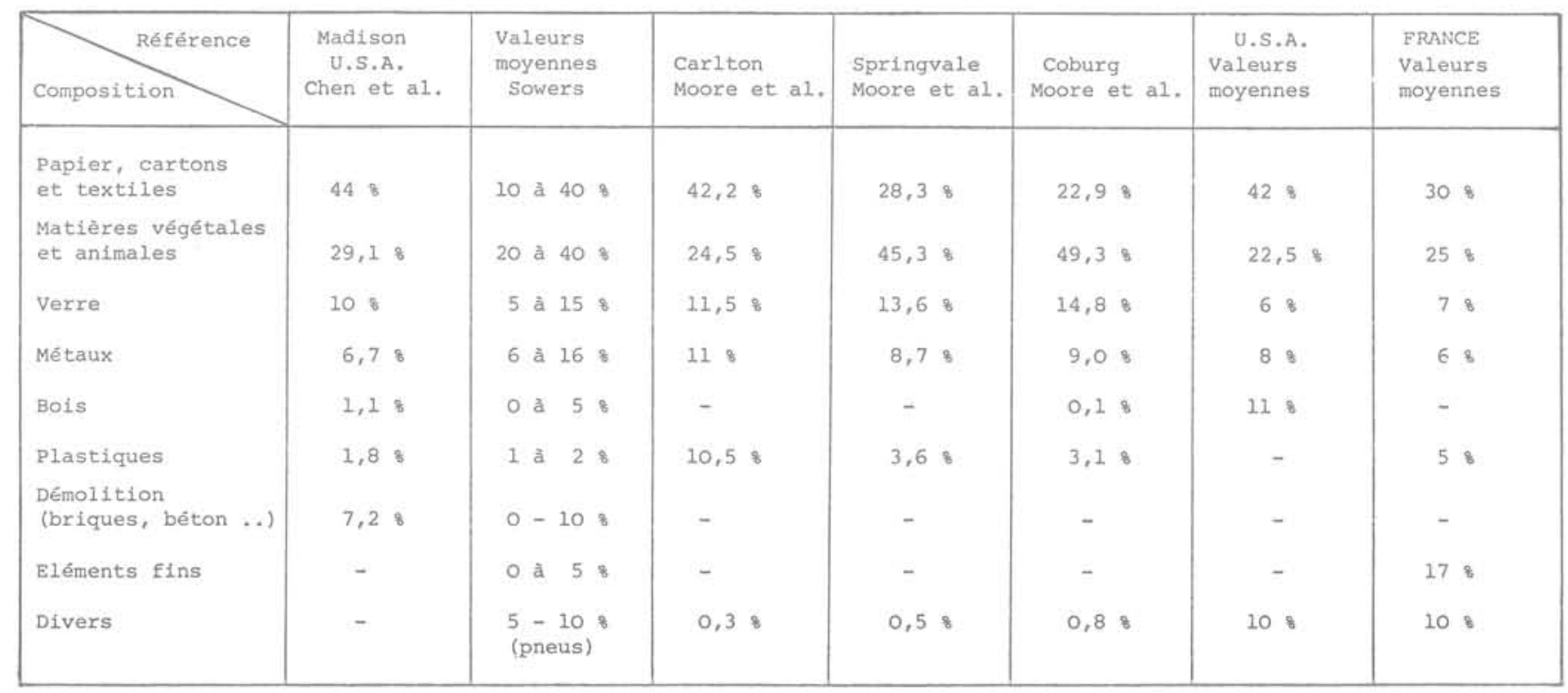

Tableau I - Comparaison de la composition de quelques décharges municipales 
La particularité essentielle des décharges de déchets ménagers réside dans la décomposition lente due aux actions ohysico-chimiques (infiltrations d'eau, oxydations, entrainement de particules ...) et à l'activité biochimique qui est continue et se traduit par la présence de liquides et de gaz. La nroduction de méthane ( 35 à 45 \%), de gaz carbonique $(35 \%)$, et d'azote ( 15 à $25 \%$ ) provient essentiellement de la décomposition des déchets animaux et végétaux : on estime généralement que les dégagements de méthane (dont la concentration crô̂t avec le temos) neuvent durer

20 ans. Ces actions s'accompagnent d'une élévation de la température des déchets qui est par conséquent indicatrice de l'activité de la décharge : on note des valeurs moyennes comprises entre 35 et $45^{\circ} \mathrm{C}$.

\section{COMPORTEMENT GEOTECHNIOQUE DES DECHARGES}

La caractérisation des résidus, qui constitue une étape indispensable pour la orévision du comportement d'une décharge, est bien olus délicate que celle des sols du fait de la grande hétérogénéité du matériau et de la présence d'éléments résistants. Dès la reconnaissance, les sondages sont souvent difficiles à réaliser et les quantités de matériaux récupérés au carottage sont faibles, notamment sous la nappe. Le battage d'un carottier de petit diamètre et la réalisation de puits d'observation semblent être les meilleurs techniques. Sowers (1968) propose de caractériser la résistance globale à l'aide de l'essai standard de pénétration (SPT) ce qui est à rapprocher des essais au nénétromètre réalisés aussi bien à Roanne qu'à Arnouville. Dans les deux cas, on enregistre quelques Dics corresnondant à des éléments durs ou à des pneus, mais l'allure générale des courbes reste significative. La détermination de modules d'Young a également été tentée oar Moore (1977) à l'aide d'essais à la plaque mais les résultats semblent assez peu significatifs pour les problèmes à long terme spécifiques aux déchets.

La détermination de paramètres géotechniques en laboratoire est a priori délicate du fait de la représentativité đouteuse des échantillons et du comportement différé du matériau. Rao (1977) et Chen (1977) ont cependant développé des oedomètres de très gros diamètres permettant d'appliquer une contre pression, de contrôler le drainage, de orélever des gaz et de recueillir des liquides polluants. Les auteurs s'accordent toutefois pour reconnaitre que ces essais ont une valeur théorique et que l'essai de chargement en vraie grandeur reste une solution mieux adaptée.

\section{Capacité portante :}

La capacité portante des ordures ménagères, c'est-à-dire leur aptitude à sunporter des charges, est généralement faible. Sowers (1968) montre que la croute de surface joue un rôle important. Toutefois, son épaisseur est limitée et sous ce niveau on ne peut quère combter que sur une capacité portante comorise entre 25 et
$100 \mathrm{kPa}$. En règle générale, on essaiera de compacter au mieux la surface et on se limitera à 50 à $80 \mathrm{kPa}$ pour éviter toute rupture.

\section{Tassements :}

Plusieurs mécanismes sont responsables du tassement des résidus ménađ̣ers (Sowers, 1973) :

- Action mécanique : la distorsion et la réorientation des divers composants produisent une consolidation similaire à celle des sols organiques.

- Percolation et ravinements : l'eau entraine les fines vers les vides laissés par les gros éléments.

- Changements ohysico-chimiques : il s'agit essentiellement de la corrosion, de l'oxydation et de la combustion.

- Décomposition biochimique : la fermentation et la décomposition des matières végétales et animales sont des phénomènes continus dans le temos.

- Intéractions : ces diverses actions interfèrent entre elles pour favoriser le phénomène et générer de nouveaux facteurs de décomnosition.

Le tassement qui se traduit par l'expulsion de liquides et de gaz ne cesse quasiment jamais et c'est la phase dite de compression secondaire qui est généralement prépondérante. La vitesse de ces tassements dépend essentiellement de la comnacité, de la teneur en eau, de la temnérature et des conditions d'environnement, mais c'est toutefois durant la première année que se produit une grande partie des ohénomènes. La diminution d'énaisseur sous poids propre neut ainsi atteindre 10 à $30 \div$.
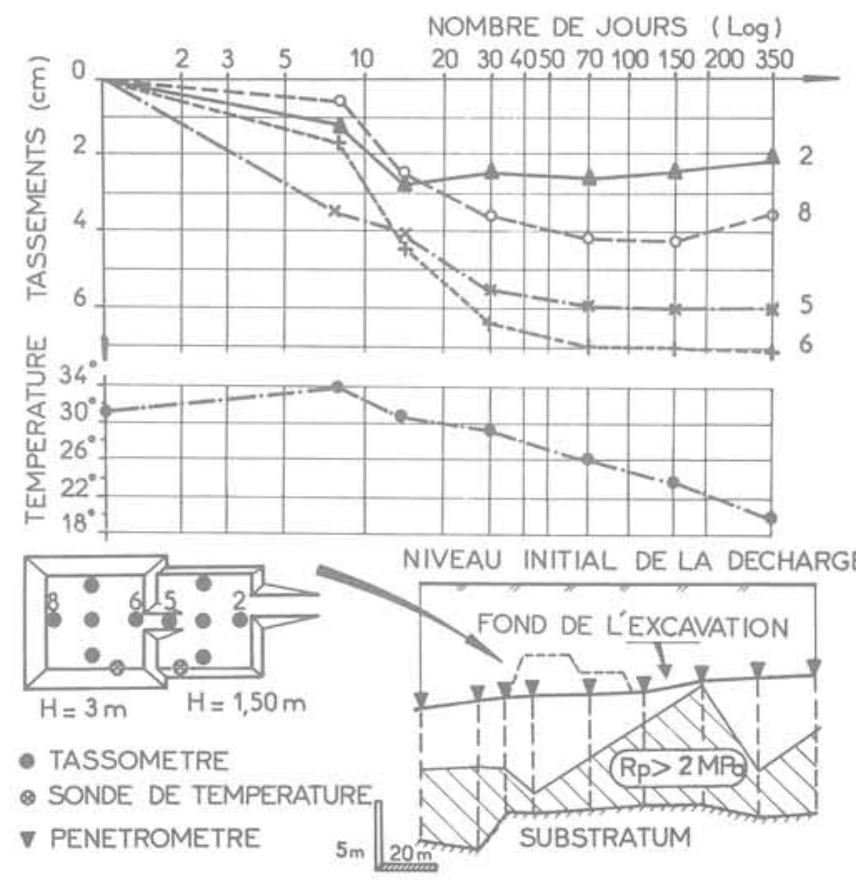

Fig.2 La décharge de Roanne: Instrumentation et résultats 
Le tassement des résidus ménagers est souvent supposé similaire à celui des sols organiques. Cela conduit Sowers (1973) à décomposer lè nhénomène en deux parties :

- Une phase primaire qui dure rarement olus d'un mois et pendant laquelle l'indice des vides décroît suivant la relation bien connue :

$$
\Delta \mathrm{e}=-C_{c} \cdot \log \frac{\sigma_{0}^{\prime}+\Delta \sigma}{\sigma_{0}^{\prime}}
$$

où $\sigma:$ est la contrainte effective initiale et $\quad \Delta \sigma \quad I^{\prime a c c r o i s s e m e n t}$ de contrainte dû à un chargement. L'indice de combression $C_{C}$ est donné par la figure 3 en fonction de l'indice des vides initial. Moore et Pedler (1977) ont confirmé le fuseau donné par Sowers à l'aide d'essais de chargement à la plaque sur une décharge contrôlée. Ces résultats, obtenus en supposant une densité des matières solides de 2, sont tout à fait concordants.

- Une phase secondaire qui résulte de la compression secondaire, de l'action physicochimique et de la décomposition biochimique et qui se traduit par la relation :

$$
\Delta e=-\alpha . \log \frac{t_{2}}{t_{1}}
$$

entre l'indice des vides et le temos. Le coefficient de compression secondaire $\alpha$ est donné par la figure 4 suivant les conditions de la décomoosition.

Ces dernières valeurs sont généralement considérées pessimistes. Keene (1977) a réalisé des mesures de tassement à diverses profondeurs d'une décharge. Cela conduit à une valeur moyenne du coefficient $\alpha$ qui est assez proche de la limite "conditions non favorables à la đécomposition". Ceci est confirmé par Yen et Scanlon (1975) sur la base de comparaisons de vitesses relatives de tassements de trois décharges contrôlées dans la région sèche de Los Angeles.

Les valeurs de Sowers semblent donc pouvoir être utilisées pour des projets, bien que les paramètres à introduire (conditions de la décomoosition, indice des vides, pourcentage de matières organiques) paraissent bien délicats à estimer avec précision. On peut également reprocher à ce modèle qu'ilsoit calqué sur le comportement des sols, alors que la plupart des hypothèses habituelles ne sont pas satisfaites. En effet, on n'a vraisemblablement pas la saturation complète (orésence de gaz), les grains et le liquide interstitiel ne sont pas incompressibles, la validité de la loi de Darcy est très douteuse et, surtout, on a prépondérance des phénomènes secondaires. Pour tenir compte de ces effets particuliers, divers auteurs ont proposé des modèles plus sophistiqués. Zimmerman et al. (1977), notamment, ont bâti une relation entre la dissination de pression interstitielle et le temps qui se présente sous forme d'une équation de continuité incluant l'influence des déformations, de l'activité chimique et biologique et de la variation du degré de saturation avec le temps.
Les essais de consolidation en laboratoire, réalisés par Rao (1977) puis chen et al. (1977), ont montré une bonne concordance entre les mesures et les prédictions faites suivant ce type de modèle. Toutefois, l'allure des courbes de tassements mesurés en laboratoire est très différente de ce que l'on obtient en général in-situ et la distribution en fonction du temps que l'on peut calculer dépend essentiellement de la théorie choisie pour tenir comnte des effets secondaires. A l'évidence, de nouvelles recherches, aussi bien en laboratoire qu'in-situ, semblent nécessaires si l'on veut développer des méthodes de prédiction des tassements et affiner les ordres de grandeur des paramètres.

\section{PROBLEMES LIES A L'ACTIVITE BIOCHIMIQUE}

Le méthane qui se dégage des décharges peut s'accumuler sous des fondations ou dans des vides importants, ce qui est dangereux quand on réalise des excavations ou des sondages (formation de grisou). Des explosions ou des feux avec mort d'hommes ou dégats matériels imnortants ont ainsi été cités dans la littérature spécialisée, sans parler des maladies ou des cas d'asnhyxies. Sachant de olus qu'une nappe de gaz peut se propager sur des distances de 1 'ordre de $200 \mathrm{~m}$, l'intérêt de mesures de contrôle apbaraît évident.

Afin d'éviter ces problèmes, le géotechnicien doit proposer des dispositions permettant d'emprisonner les gaz ou de les ventiler. On a en général recours à des tranchées filtrantes remblies de graviers ou à des tuyaux d'aération équipés de matériaux résistants aux sulfates et pouvant admettre des déformations différentielles. Dans certains cas, ces systèmes ont été conçus afin de récunérer l'énergie combustible qui est parfois importante puisqu'il est courant de constater des débits de 20 à $80 \mathrm{~m} 3 / \mathrm{h}$ dans des forages. L'utilisation de membranes est également envisageable mais n'est oas toujours compatible avec les grandes déformations.

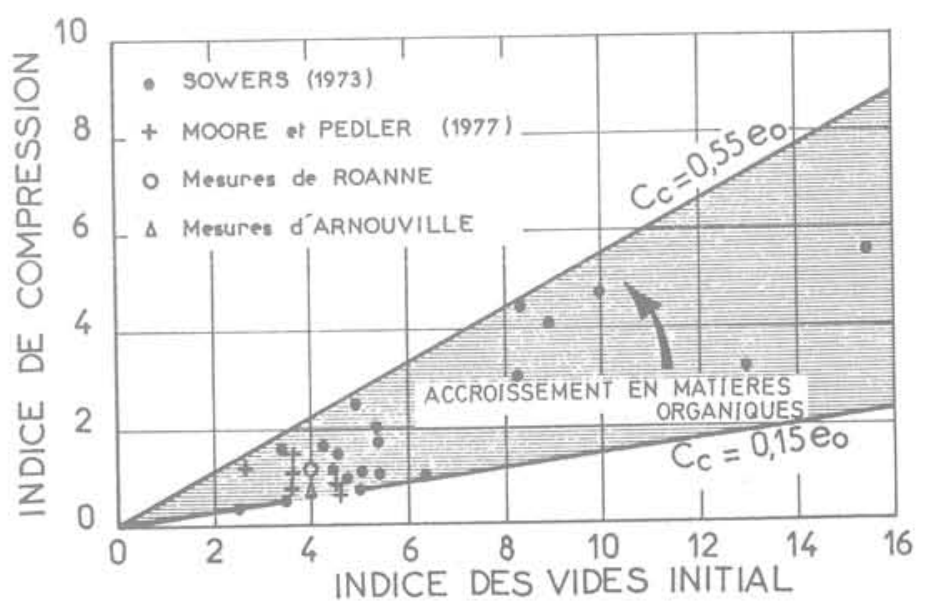

Fig. 3 Indice de compression en fonction de l'indice des vides 


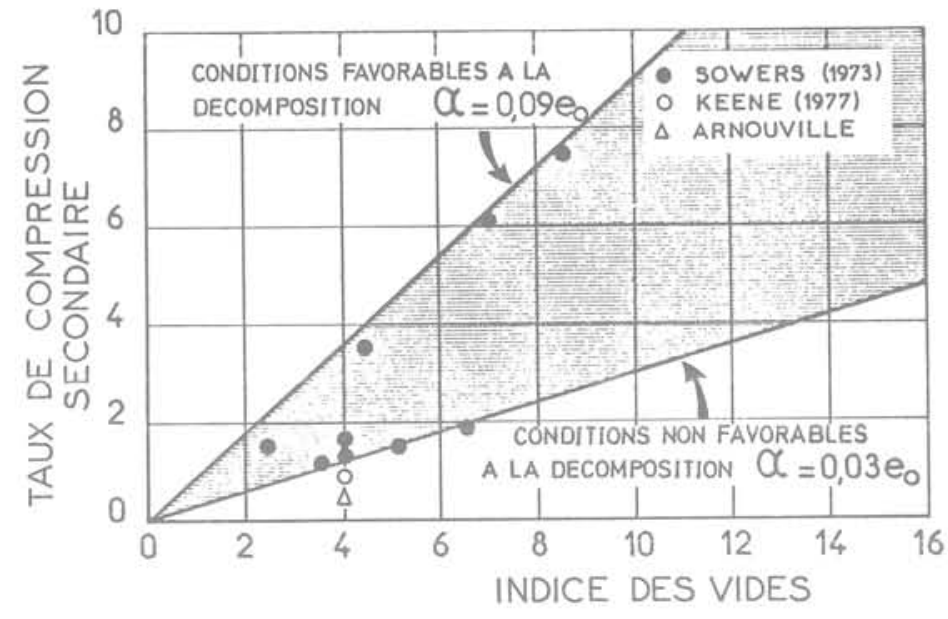

Fig. 4 Taux de compression secondaire en fonction de l'indice des vides

Lả décomposition prođuit également des acides organiques qui attaquent les tuyaux d'acier, les drains, les fondations de bâtiments. Un tuyau d'acier galvanisé peut, en effet, être piqué en un an s'il n'est pas traité avec un revêtement résistant à la corrosion. C'est également le cas du ciment qui est attaqué par 1'ion sulfate.

Les problèmes de pollution du sous-sol par les liquides s'écoulant de la décharge nécessitent, bien entendu, d'être évalués en même temos que les aspects géotechniques.

\section{AMELIORATION ET TRAITEMENT DES RESIDUS}

Les problèmes liés à la faible capacité portante et aux tassements importants qui caractérisent les décharges peuvent être diminués par le traitement ou l'amélioration des résidus. Diverses techniques sont envisageables.

Compactage : L'augmentation de la densité d'une décharge permet de diminuer son volume, de réduire les vides et d'uniformiser le matériau. C'est donc une opération qu'il faut recommander mais qui demande un matériel snécial permettant de niveler et de broyer les résidus. Le résultat qui dépend de l'éoaisseur des couches compactées et de la teneur en eau lors du compactage est ontimal durant les 8 à 12 premières passes et limité en profondeur à environ 3 mètres. C'est donc une technique qui doit être réservée aux décharges en cours de construction et qui est assez neu efficace pour traiter les sites existants.

Préchargement : Cette méthode qui consiste à placer une charge sur le terraín jusqu'à ce que la vitesse de tassement soit compatible avec les critères de l'ouvrage à construire est classique et efficace pour les sols. Pour les résidus, les essais à la plaque de Moore et Pedler (1977) ont montré l'influence positive d'un préchargement sur la vitesse et la valeur des tassements à court terme. Tnute- fois, leur amolitude finale est gouvernée par le comportement à long terme et il subsiste un doute quant à l'efficacité du préchargement sur la comoression secondaire.

Injections : Rao (1977) a réalisé des essais en laboratoire et en cuve sur des résidus traités aux cendres volantes, elles-mêmes intéressantes à réutiliser. Les résultats sont toutefois encore insuffisants et de nouvelles recherches seraient souhaitables ainsi que sur les injections de produits accélérant la décomnosition (mélange d'azote, de phosphore et de potassium).

\section{MESURES REALISEES SUR LA DECHARGE CONTROLEE D'ARNOUVILLE}

La décharge contrôlée d'Arnouville-les-Mantes est exploitée depuis quelques années selon la technique des casiers : les déchets, uniquement ménagers, sont répandus et compactés en couches minces d'environ $50 \mathrm{~cm}$ d'épaisseur. Le casier $n^{\circ} 3$, dont I'énaisseur est en moyenne de $15 \mathrm{~m}$, a été équioé d'un profil de tassomètres type LPC (fig.l), destiné à contrôler les tassements des résidus qui sont actuellement dans un stade actif de la décomposition si l'on en croit la température élevée $\left(40\right.$ à $50^{\circ} \mathrm{C}$ ) et $l^{\prime}$ importance des dégagements gazeux dans des puits busés pour capter le méthane. Le chargement du casier a été effectué progressivement oar un remblaiement d'argile à meulière et a provoqué les tassements reportés en fonction du logarithme du temos sur la figure 1. $\mathrm{Si}$ I'on considère, selon les indications de Sowers, que la phase primaire cesse au bout d'environ trois mois, on peut calculer une valeur moyenne du taux de compression secondaire sur les derniers 400 jours. Pour un indice des vides initial de 4 on obtient une valeur inférieure à la borne $\alpha=0,03$ e donnée par Sowers (fig. 4). Toutefois, ${ }^{\circ}$ la décombosition actuelle s'effectue vraisemblablement en conditions anaérobies et l'indice des vides pourrait être inférieur à 4 si l'on en juge par le comnactage énergique et le répandage systématique de couches de sable au sein des ordures.

Les essais au pénétromètre statique réalisés sur le casier ont donné des valeurs assez homogènes, si on exclue systématiquement les pics correspondant à des points durs. La résistance de pointe moyenne est de 5000 à 6000 $\mathrm{kPa}$ et varie peu avec la orofondeur, contrairement au frottement latéral qui crôt de façon continue.

Un effort de suivi de ce site parait souhaitable vis-à-vis des tassements (afin de confirmer les mesures actuelles et de voir si une stabilisation peut être obtenue avec le temns) ainsi que vis à vis de la résistance et notamment de son évolution en fonction de la densité et du tembs. 


\section{LES REMBLAIS D'ESSAI DE ROANNE}

Deux remblais expérimentaux de 1,50 et $3 \mathrm{~m}$ d'épaisseur ont été réalisés sur la décharqe municipale de la ville de Roanne (fig. 2), afin d'évaluer les tassements prévisibles lors de la construction d'une plateforme autoroutière. La reconnaissance au pénétromètre dynamique réalisée après excavation de $7 \mathrm{~m}$ de résidus a permis de vérifier une certaine homogénéité d'ensemble et de mettre en évidence une zone de moindre résistance $(\mathrm{Rp}<2000 \mathrm{kPa})$ sur laquelle a été implantée la planche expérimentale.

Le nivellement de la surface de la décharge a montré des mouvements inférieurs à un centimètre sur deux ans et demi, ce qui indique que la compression secondaire est négligeable. Ceci a été confirmé par les mesures de tassomètres sous le chargement des remblais, puisque le tassement primaires'est produit en moins de deux mois et qu'après 350 jours les mouvements sont très faibles (fig. 2). Ces observations sont à rapprocher des mesures de la temoérature au sein des ordures qui indiquent une relance de l'activité biochimique (certainement aérobie)dans les jours qui ont suivi l'excavation $\left(\theta=34^{\circ}\right)$, puis une constante diminution atteignant $15^{\circ} \mathrm{C}$ en un an. Cette stabilisation de la décharge est vraisemblablement due à son âce (l'exoloitation a démarré vers 1910) et à une proportion importante de déchets non ménagers et donc moins putrescibles.

Le calage de l'indice de compression $C_{C}$ sur les mesures de tassement, effectué en ${ }_{C}$ considérant un indice des vides e de 4 , donne une valeur moyenne tout à fait concordante avec les valeurs de Sowers (fig. 3). Notons que ce comportement satisfaisant a permis d'envisager la réalisation de la plateforme autoroutière avec une chaussée souple capable d'absorber les quelques mouvements différentiels prévisibles.

\section{CONCLUSION}

Le stockage des résidus de l'usage domestique est devenu un problème économique suffisamment crucial pour que l'on cherche à réutiliser les décharges dans le cadre de zones d'aménaquement ou de constructions. Le aéotechnicien et le projeteur sont alors confrontés aux problèmes habituels pour les sols de faibles caractéristiques, mais doivent également compter avec la furte hétérogénéité des matériaux, l'activité due aux actions physico-chimiques et biochimiques ainsi qu'avec les déformations imoortantes et non uniformes.

La synthèse des méthodes proposées jusqu'à nos jours pour prévoir l'ordre de orandeur des phénomènes constitue certainement une base acceptable pour les études ainsi que l'on a pu le vérifier sur deux sites expérimentaux suivis par les Laboratoires des Ponts et Chaussées. Toutefois, les mesures restent dispersées et les prédictions dépendent essentiellement de la détermination, toujours délicate, de paramètres comme l'indice des vides.
A l'évidence, de olus amples recherches seraient utiles et, en l'état actuel, il semble prudent d'avoir recours à des planches expérimentales pour pallier ces difficultés.

Le suivi des remblais expérimentaux de Roanne a été réalisé avec le concours de MM. Perrin et Bargillat du L.R.P.C. de Lyon, et les mesures sur la décharge d'Arnouville ont été assurées par M. Baldit du L.R.O.P. de Trappes.

\section{REFERENCES}

Cartier G., Long N.T., Pouget P., Bargillat R. et Cudennec J.P., (1981)

Déchets urbains et pneumatiques usaqés en aénie civil. - C.R. loè CIMSTF, STOCKHOLM

Chen W.H., Zimmerman R.E. et Franklin A.G., (1977) -

Time settlement characteristics of milled urban refuse. - Soec. Conf. of the GED, ASCE, Univ, of Michigan, 136 - 152

Cudennec J.P., Gican J.P., Cartier G., (1981) Constructions de remblais autoroutiers sur ballastières remblayées. IIème Journées nationales qéotechniques, Nantes.

Keene P., (1977) Sanitary landfill treatment, Interstate highway 84 - Spec. conf. of the GED, ASCE, Univ. of Michigan, 632 - 644

Moore P.J. et pedler I.V., (1977) Some measurements of comnressibility of sanitary landfill material. C.R. 9ème CIMSTF, Spec. session $n^{\circ} 11,319-330$, Tokyo.

Rao S.K., Moulton L.K. et Seals .R.K., (1977) Settlement of refuse landfills. Spec. conf. of the GED, ASCE, Univ. of Michigan, 574598

Sowers G.F., (1973) settlement of waste disnosal fills. C.R. 8 è̀me CIMSTF, (2), 207 - 210, Moscou

Yen V.C. et Scanlon B., (1975) Sanitary landfill settlement rates. Proc. ASCE, J.of the GED, (101), GT 5, 475 - 487 .

Sowers G.F., (1968) Foundation problems in sanitary landfills. Proc. ASCE, J. of the SED, (94), SAl, $103-116$ 